\title{
Compound Heterozygous Splicing Variants In KIAA0586 Cause Fetal Short-Rib Thoracic Dysplasia And Cerebellar Malformation: The Use of Whole Exome Sequencing In Prenatal Diagnosis
}

Qianying Zhao ( $\square$ ofeizhaoqy@163.com )

Department of Obstetrics \& Gynecology

\section{Bocheng Xu}

Department of Obstetrics \& Gynecology

\section{Qinqin Xiang}

Department of Obstetrics \& Gynecology

Yu Tan

Department of Obstetrics \& Gynecology

Hanbin Xie

Department of Obstetrics \& Gynecology

\section{Qianqian Gao}

Department of Ultrasound

\section{Lingyi Wen}

University Hospital, Sichuan University

\section{He Wang}

Women and Children (Sichuan University)

\section{Mei Yang}

Department of Obstetrics \& Gynecology

Shanling Liu

Department of Obstetrics \& Gynecology https://orcid.org/0000-0001-8788-7991

\section{Research Article}

Keywords: Short-rib thoracic dysplasia, Joubert syndrome, Prenatal diagnosis, KIAA0586, Splicing variants, Whole exome sequencing

Posted Date: September 21st, 2021

DOl: https://doi.org/10.21203/rs.3.rs-886998/v1 
License: (c) (i) This work is licensed under a Creative Commons Attribution 4.0 International License. Read Full License 


\section{Abstract}

Background: Short-rib thoracic dysplasia (SRTD) and Joubert syndrome (JS) are rare genetic ciliopathies, both patients can manifest cerebellar malformation and variable developmental delays. However, neither could be easily diagnosed during pregnancy due to limited fetal phenotype. Here, we investigated a fetus with short limbs, polydactyly initially and uncovered a compound heterozygous pathogenesis through whole exome sequencing (WES).

Results: Merely short limbs and polydactyly of the fetus were detected during second trimester of gestation. Two variants (c.3940+1G>A and c.3303G>A), affecting splicing of KIAA0586 gene, were identified from amniocytes through WES. The presence and effect of these mutations were further validated on DNA and RNA level through Sanger sequencing. More intensive fetal monitoring was applied; deformed cerebellar malformation and restricted thoracic cage of the fetus were additionally uncovered.

Conclusion: Herein, we discovered a genetic pathogenesis of KIAA0586 gene associated with SRTD and/or JS in a fetus with mild ultrasound anomalies initially. With the information of prenatal WES and distinct phenotypes of the fetus uncovered by imaging examination, we could reach more accurate clinical diagnosis and provide valuable prognosis information for parents.

\section{Background}

Short-rib thoracic dysplasia (SRTD) syndromes are a group of autosomal recessive or digenic recessive skeletal ciliopathies characterized by short ribs, short limbs and restricted thoracic cage. Polydactyly is variably present, non-skeletal involvement can include anomalies of major organs such as the brain, heart, kidneys, liver, pancreas, intestines and genitalia. SRTDs encompass asphyxiating thoracic dystrophy (ATD), short rib-polydactyly syndrome (SRPS) and etc [1]. Some types of SRTDs are lethal in the neonatal period due to respiratory insufficiency secondary to a severely restricted thoracic cage, whereas others are compatible with life. Given to the genetic heterogeneity, SRTDs have yet been classified into 20 types (SRTD1 20), caused by mutations in genes such as IFT80, DYNC2H1, TTC21B, WDR19, NEK1, WDR35, WDR60, IFT140, IFT172, WDR34, CEP120, KIAA0586, DYNC2LI1, IFT52, TCTEX1D2, IFT43, IFT81 and INTU gene[2-4].

Joubert syndrome (JS) is a neurodevelopmental disorder characterized by a distinctive cerebellar and brain stem malformation called the molar tooth sign (MTS), hypotonia and variable developmental delays. Additional findings occur in JS patients include retinal dystrophy, respiratory control disturbances, renal disease, ocular colobomas, occipital encephalocele, hepatic fibrosis, polydactyly, oral hamartomas, and endocrine abnormalities [5,6]. Predominantly, JS is inherited in an autosomal recessive manner, but $X$-linked and digenic inheritance have also been reported. JS demonstrates extreme phenotypic variability and genetic heterogeneity [7]. To date pathogenic variants in more than 30 genes are known to cause JS. 
A molecular diagnosis of JS can be established in about $62 \%-94 \%$ of individuals with a clinical diagnosis of JS [8].

Here, we present a prenatal case of fetal with short limbs and polydactyly. A novel splicing and a known synonymous mutation of KIAA0586 gene (OMIM: 610178) were detected in amniocytes by whole exome sequencing (WES). The mutations were inherited from the fetal parents respectively, establishing a compound heterozygous pathogenesis. Interestingly, though one of which is synonymous, both mutations were proved to affect splicing of KIAA0586 gene, which has been associated with SRTD (type 14) or JS.

\section{Clinical Summary}

A primigravid woman was referred for genetic counseling during second trimester, because of abnormal fetal ultrasound findings: polydactyly, short limbs and persistent left superior vena cava (PLSVC). The ultrasound examination at $24+1$ weeks of gestation revealed polydactyly of the fetal right hand, a fetal femur length $(\mathrm{FL})$ of $3.9 \mathrm{~cm}(\sim 22+5 \mathrm{w})$. PLSVC with dilated coronary sinus was detected by fetal echocardiography.

The woman and her husband were non-consanguineous, and there was no family history of congenital malformations or genetic diseases. The pedigree of the family is shown in Fig. 1. Amniocentesis was performed at $25+5$ weeks of gestation, and DNA extracted from the amniocytes was tested by WES and chromosome microarray analysis (CMA).

Further imaging monitoring was recommended according to genetic mutations found by WES, including serial fetal sonography and the axial brain magnetic resonance imaging (MRI). More distinct phenotypes of the fetus were uncovered, consist with genetic results.

\section{Molecular Results}

CMA was successively performed for the fetus but with negative results: no numerical abnormalities of chromosomes, copy number variants (CNVs) or regions of homozygosity were discovered. However, we identified two variants in KIAA0586 gene (NM_001244189.2) from the amniocytes in a compound heterozygous state: $c .3940+1 G>A$ and c.3303G $>$ A (p.P1101P). The presence of these mutations was further validated by Sanger sequencing (Fig. 3).

The novel canonical splice site mutation (c. $3940+1 \mathrm{G}>\mathrm{A}$ ) inherited paternally has not been reported in any database, including the Human Gene Mutation Database (HGMD), ClinVar or GnomAD databases. Our study showed that the novel canonical splice site mutation (c. $3940+1 \mathrm{G}>\mathrm{A}$ ) resulted in deletion of exon 26 and also introduced a framshift, producing a premature termination codon. Meanwhile, the synonymous mutation (c.3303G > A; p.P1101P) inherited from the pregnant woman was predicted to be deleterious, according to CADD, Splice Al and dbscSNV. To varify the underlying pathogenicity of these mutations, Sanger sequencing was performed on cDNA from the fetal parents. The results indicated that 
the synonymous mutation (c.3303G > A) led to deletion of 56 basepairs of exon 23 , resulting in a framshift with premature truncation of the protein. This result was consistent with the previous study reported in a JS patient [13] (Fig. 4).

\section{Imaging Findings}

Fetal MRI at $31+5$ weeks of gestation showed deformed cerebellar vermis and characteristic MTS of the fetus (Fig. 2a). Strikingly, hypoplastic cerebellar vermis, enlarged third ventricle, relatively small thoracic cage and short humerus length $(\mathrm{HL}, 5.03 \mathrm{~cm},-2.9 \mathrm{SD})$ besides polytactyly were later found at 33 weeks of gestation by fetal sonography (Fig. 2b-2f). Ultimately, the couple chose to terminate the pregnancy after multidisciplinary consultation due to likely poor prognosis after birth.

\section{Methods}

Experiment on human subjects was approved by the Ethical Review Board of West China Second University Hospital, Sichuan University. Genomic DNA of the family members was extracted from amniocytes and parents' peripheral blood leukocytes using QIAamp DNA Blood Mini Kit (QIAGEN). To detect the genetic factors for the fetus, CMA and trio-WES were performed using genomic DNA from the family members (II-1, II-2, III-1).

Genomic DNA (gDNA) from the fetus and parents was subjected to single nucleotide polymorphism (SNP) array with the CytoScan 750K Array (Thermo Fisher Scientific), following the manufacturer's instructions. Molecular karyotype analysis was performed using Chromosome Analysis Suite v4.1 (Thermo Fisher Scientific). The GRCh38 (hg38) genome was used for annotation. CNVs larger than 100 $\mathrm{kb}$ or those that affected more than 50 contiguous probes were considered. Genetic pathogenicity and clinical significance of CNVs were systematically evaluated according to American Society for Medical Genetics and Genomics (ACMG) guidelines [9] and previous publication [10], respectively.

Exome capture sequencing was performed using the NanoWES Human Exome V1 (Berry genomics) following the manufacturer's protocol. The enriched library was sequenced through Illunima NovaSeq6000 platform with 150-bp paired-end reads. Next, Burrows-Wheeler Aligner software tool was used for aligning the sequencing reads with GRCh38 (hg38). After that, local alignment and recalibration of base quality of the Burrows-Wheeler aligned reads was performed by the GATK Indel Realigner and the GATK Base Recalibrator, respectively (broadinstitute.org/). Then, Single-nucleotide variants (SNVs) and small insertions or deletions (InDels) were identified by GATK Unified Genotyper (broadinstitute.org/). Finally, functional annotation was performed using ANNOVAR and the Enliven Variants Annotation Interpretation System (Berry genomics). Public databases using for filtering include gnomAD (http://gnomad.broadinstitute.org/), 1000 Genomes Project (1000G) (http://browser.1000genomes.org), and etc. Pathogenicity of SNVs were evaluated based on the scientific medical literature and disease databases, such as PubMed (https://www.ncbi.nlm.nih.gov/pubmed/), ClinVar(http://www.ncbi.nlm.nih.gov/clinvar), OMIM (http://www.omim.org), Human Gene Mutation 
Database (http://www.hgmd.org), and Human Genome Variation Society (http://www.hgvs.org/dblist/dblist.html).

Candidate variants on each independent gDNA sample (II-1, II-2, III-1) were validated using Sanger sequencing: polymerase chain reaction (PCR) amplification was performed using primer pairs (Table 1) designed to cover variants identified by WES. Total RNA was extracted from the parents' fresh peripheral blood samples, using RNApure Blood Kit (CWBIO, CW0582S), and then reversed to CDNA through synthesis Kit (Thermo Scientific, K1622). Target sequences of cDNA were amplified using PCR as well, using primers designed using Primer 3 software Version 0.4.0 (http:// bioinfo.ut.ee/primer3-0.4.0/) (Table 1). Sequencing of PCR products were conducted by ABI 3500 Genetic Analyzer (Thermo Fisher Scientific) to verify the changes on both DNA and RNA level. Data were evaluated using the Chromas software (2.6.5).

\section{Table 1 Primers used in the current study}

\begin{tabular}{|lll|}
\hline Primers & Forward & Reversed \\
\hline KIAA0586 SNV & 5'ATGTCTGTGGCTAAGGATG 3' & 5'AGGGTGGGATTATGAGGAA 3' \\
$($ c.3940 + 1G > A) & & \\
\hline KIAA0586 SNV & 5'AAGCTCTTGCTGAGACCATTGC 3' & 5'AGCCCAGAACTTCAAGACCAGC 3' \\
$($ c.3303G > A) & & \\
\hline $\begin{array}{l}\text { KIAA0586 mRNA } \\
\text { (c.3940 + 1G > A) }\end{array}$ & 5'CTACTACTACACCTCCTCCA 3' & 5'TCCAAGTCCTCTGAATGATAG 3' \\
\hline KIAA0586 mRNA & 5'TTCCGCCAGTTGCTTCTA 3' & 5'GGCAGGCATATCATCTCC 3' \\
(c.3303G > A) & & \\
\hline B-actin & 5'CTGGCACCACACCTTCTACAATG 3' & 5'CCTCGTAGATGGGCACAGTGTG 3' \\
\hline
\end{tabular}

\section{Discussion}

It is recommended that laboratories offering prenatal WES report pathogenic and likely pathogenic variants, as determined using ACMG variant interpretation guidelines in known disease genes consistent with the reported fetal phenotype $[11,12]$. Through DNA and RNA validation, we proved that both variants affected splicing and might lead to frameshift of KIAA0586 gene. Therefore, the compound heterozygous mutations were rated as likely pathogenic combined with low frequency in general population. It is worth noting that the synonymous variant, at the last basepair of exon 23 , can be easily neglected while analysis. However, the substitution leads to use of a cryptic splice site in exon 23 and deletion of 56 basepairs, resulting frameshift and in premature truncation of the KIAA0586 protein [13]. Therefore, disease databases such as ClinVar or HGMD and functional researches should be queried for putative pathogenic variants [13-15]. Moreover, impact of splice-site variants was confirmed through functional 
analysis by RNA in our study. RNA verification using the parents' blood samples successfully prevented another invasive operation for the fetus and acquired rapid validation results for the family.

Recently, WES may be considered for a fetus with ultrasound anomalies after standard CMA and karyotype analysis have failed to yield a definitive diagnosis [16]. However, abnormal karyotype and CNVs can merely explain partially the genetic etiology, more than half of fetuses with sonographically identified structural anomalies remain without a diagnosis. In addition, WES is increasingly used in both pediatric and adult population, for multiple congenital anomalies, skeletal, neurodevelopmental and neuromuscular disorders [17-20]. Given the success in these patients, WES, limited to the analysis of coding regions of known genes, is currently applied prenatally. Studies reveal that WES increases the diagnostic yield in structurally abnormal fetuses by about 8-10\% after normal karyotype and CMA results, and the detection rate is strongly proportional to the severity of phenotype [21, 22]. For our case, multiple fetal anomalies demonstrated a strong indication to implement prenatal WES. Instead of sequential testing, CMA and WES were simultaneously suggested and performed due to late gestation age, leading to a timely and comprehensive genetic diagnosis for the fetus.

Given the analysis of WES is phenotype driven; prenatal reporting of variants is of great challenge due to limited and ambiguous fetal imaging findings. Accurate identification of fetal structural anomalies is sophisticated by gestation age, fetal position, the ultrasound equipment, and the experience of clinicians [16]. The diagnostic yield rate of WES is strongly correlated with numbers of fetal anomalies and specificity of phenotypes [23]. Moreover, adequate clinical information, including reproductive history, family history and parental consanguinity, should be required to generate the most accurate interpretation of genetic results. Through routine scheme of antenatal care, fetal cerebral MRI would not have been arranged. Hence, MTS, the typical manifestation of JS, might have been undiscovered for the fetus. At 24 + weeks of gestation, the fetal femur length was merely one week shorter compared to fetuses of similar gestational age. Thanks to more intensive monitoring, fetal humerus length was found markedly shorter (-2.9SD), as well as small thoracic cage and cerebellar vermis revealed at 33 weeks of gestation by diagnostic ultrasound.

Due to genetic diagnosis during pregnancy, more tailored and frequent prenatal care (e.g. sonography and MRI) was implemented for the fetus. Consequently, the health-care professionals from multidisciplinary provided collectively clinical and genetic results for the couple. The genetic results can not only assist in determining the fetal prognosis, but also indicate the recurrence risk. According to authoritative databases such as OMIM, patients with KIAA0586 mutations might manifest JS or SRTD (type 14). Moreover, neurodevelopmental disorders, not detectable with fetal imaging, may onset after birth. The couple's offspring has a likelihood of $25 \%$ to possess a compound heterozygous genotype, leading to diseases. Subsequently, informed genetic counseling about future reproductive options, including preimplantation genetic testing or diagnostic prenatal testing, could be addressed.

\section{Conclusions}


In our study, we discovered a compound heterozygous pathogenesis of KIAA0586 gene for a fetus with short limb and polydactyly initially. We reported a novel KIAA0586 variant which expands the spectrum of KIAA0586 in JS and/or SRTDs. Depending on validated WES results, more distinct fetal phenotypes including thoracic dysplasia and MTS were timely uncovered, leading to consistency of genetic and clinical diagnosis during pregnancy. Consequently, valuable prognosis information and future reproductive alternatives were provided for the parents.

\section{Abbreviations}

SRTD: Short-rib thoracic dysplasia; ATD: Asphyxiating thoracic dystrophy; SRPS: Short rib-polydactyly syndrome; JS: Joubert Syndrome; MTS: Molar tooth sign; WES: Whole exome sequencing; PLSVC: persistent left superior vena cava; FL: femur length; CMA: Chromosome microarray analysis; MRI: Magnetic resonance imaging; HL: humerus length; SNV: Single-nucleotide variant; PCR: Polymerase chain reaction; SNP: Single nucleotide polymorphism; CNV: Copy number variant; ACMG: American Society for Medical Genetics and Genomics.

\section{Declarations}

\section{Acknowledgements}

We sincerely thank the patient and his family for their interest and cooperation.

\section{Authors' contributions}

Qianying Zhao and Mei Yang designed the study experiments. Qianying Zhao collected the data and conducted the clinical evaluations. Hanbing Xie, Bocheng Xu, Yu Tan and Qinqin Xiang performed the experiment. Qianying Zhao and Mei Yang wrote the article. Shanling Liu supervised the study experiments. All authors revised and approved the article.

\section{Funding}

This work was supported by The National Key Research and Development Program of China (2021YFC1005303) and The Program of Science and Technology Department of Sichuan Province (No.2021YFS0078).

\section{Availability of data and materials}

The data that support the findings of this study are available from the corresponding author upon reasonable request.

\section{Ethics approval and consent to participate}


Experiment on human subjects was approved by the Ethical Review Board of West China Second University Hospital, Sichuan University.

Informed consent for participation to this study was obtained from all individuals.

\section{Consent for publication}

Publication of data was informed consent to all individuals involved in this study.

\section{Competing interests}

The authors declare no conflict of interests.

\section{References}

1. Caroline Alby K, Piquand C, Huber A, Megarbane' A, Ichkou M, Legendre et al (2015) Mutations in KIAA0586 Cause Lethal Ciliopathies Ranging from a Hydrolethalus Phenotype to Short-Rib Polydactyly Syndrome. The American Journal of Human Genetics 97:1-8

2. Chen C-P, Shih J-C, Tzen C-Y, Lin Y-H, Wang W (2005) Recurrent short-rib polydactyly syndrome: prenatal three-dimensional ultrasound findings and associations with congenital high airway obstruction and pyelectasia. Prenat Diagn 25:417-418

3. Chen C-P, Chang T-Y, Chen C-Y, Wang Y-T, Tsai F-J, Wu P-C et al (2012) Short ribpolydactyly syndrome type II (Majewski): prenatal diagnosis, perinatal imaging findings and molecular analysis of the NEK1 gene. Taiwan J Obstet Gynecol 51:100-105

4. Chen C-P, Chern S-R, Chang T-Y, Su Y-N, Chen Y-Y, Su J-W et al (2012) Prenatal diagnosis and molecular genetic analysis of short rib-polydactyly syndrome type III (Verma-Naumoff) in a secondtrimester fetus with a homozygous splice site mutation in intron 4 in the NEK1 gene. Taiwan J Obstet Gynecol 51:266-270

5. Brancati F, Dallapiccola B, Valente E (2010) Joubert Syndrome and related disorders. Orphanet J Rare Dis 5(1):20

6. Poretti A, Boltshauser E, Valente EM (2014) The Molar Tooth Sign is Pathognomonic for Joubert Syndrome! Pediatr Neurol 50(6):e15

7. Bachmann-Gagescu R, Dempsey JC, Phelps IG, O'Roak BJ, Knutzen DM, Rue TC et al (2015) Joubert syndrome: A model for untangling recessive disorders with extreme genetic heterogeneity. $\mathrm{J}$ Med Genet 52(8):514-522

8. Parisi MA, Glass I Joubert syndrome and related disorders 2003. Available at: http://www.ncbi.nlm.nih.gov/books/NBK1325/

9. Riggs ER, Andersen EF, Cherry AM, Kantarci S, Kearney H, Patel A et al (2020) Technical standards for the interpretation and reporting of constitutional copy-number variants: a joint consensus 
recommendation of the American College of Medical Genetics and Genomics (ACMG) and the Clinical Genome Resource (ClinGen). Genet Med 22(2):245-257

10. Ting Hu, Zhang Z, Wang J, Li Q, Zhu H, Lai Y et al (2019) Prenatal diagnosis of chromosomal aberrations by chromosomal microarray analysis in fetuses with ultrasound anomalies in the urinary system. Prenat Diagn 39(12):1096-1106

11. Strande NT, Riggs ER, Buchanan AH, Birsoy OC, DiStefano M, Dwight SS et al (2017) Evaluating the clinical validity of gene-disease associations: an evidence-based framework developed by the Clinical Genome Resource. Am J Hum Genet 100:895-906

12. Richards S, Aziz N, Bale S, Bick D, Das S, Gastier-Foster J et al (2015) Standards and guidelines for the interpretation of sequence variants: a joint consensus recommendation of the American College of Medical Genetics and Genomics and the Association for Molecular Pathology. Genet Med 17:405-424

13. Ruxandra Bachmann-Gagescu, Phelps IG, Dempsey JC, Vivek A, Sharma, Gisele E, Ishak et al (2015) KIAA0586 is mutated in Joubert syndrome. Hum Mutat 36(9): 831-835

14. Sue Richards N, Aziz S, Bale D, Bick S, Das J, Gastier-Foster et al (2015) Standards and guidelines for the interpretation of sequence variants: a joint consensus recommendation of the American College of Medical Genetics and Genomics and the Association for Molecular Pathology. Genet Med 17(5):405-424

15. Ahmad NA, Tayoun T, Pesaran, Marina T, DiStefano A, Oza HL, Rehm, Leslie G, Biesecker et al (2018) Recommendations for interpreting the loss of function PVS1 ACMG/AMPvariant criterion. Hum Mutat 39(11):1517-1524

16. Kristin G, Monaghan NT, Leach D, Pekarek P, Prasad, Nancy C, Rose, ACMG Professional Practice and Guidelines Committee (2020) The use of fetal exome sequencing in prenatal diagnosis: a points to consider document of the American College of Medical Genetics and Genomics (ACMG). Genet Med 22(4):675-680

17. Retterer K, Juusola J, Cho MT, Vitazka FM, Glibellini F, Vertino-Bell A,et al (2016) Clinical application of whole-exome sequencing across clinical indications. Genet Med 18:696-704

18. Cukier HN, Dueker ND, Slifer SH, Lee JM, Whitehead PL, Lalanne E et al (2014) Exome sequencing of extended families with autism reveals genes shared across neurodevelopmental and neuropsychiatric disorders. Mol Autism 5:1

19. Shen T, Pajaro-Van de Stadt SH, Yeat NC, Lin JC (2015) Clinical applications of next generation sequencing in cancer: from panels, to exomes, to genomes. Front Genet 6:1-9

20. Jennifer E, Posey JA, Rosenfeld RA, James M, Bainbridge Z, Niu X, Wang et al (2016) Molecular diagnostic experience of whole-exome sequencing in adult patients. Genet Med 18(7):678-685

21. Petrovski S, Aggarwal V, Giordano JL, Stosic M, Wou K, Bier L et al (2019) Whole-exome sequencing in the evaluation of fetal structural anomalies: a prospective cohort study. Lancet 393:758-767

22. Lord J, McMullan DJ, Eberhardt RY, Rinck G, Hamilton SJ, Jones EQ et al (2019) Prenatal exome sequencing analysis in fetal structural anomalies detected by ultrasonography (PAGE): a cohort 
study. Lancet 393:747-757

23. Zhang S, Lei C, Wu J, Sun H, Yang Y, Zhang Y et al (2017) A retrospective study of cytogenetic results from amniotic fluid in 5328 fetuses with abnormal obstetric sonographic findings. J Ultrasound Med 36:1809-1817

Figures

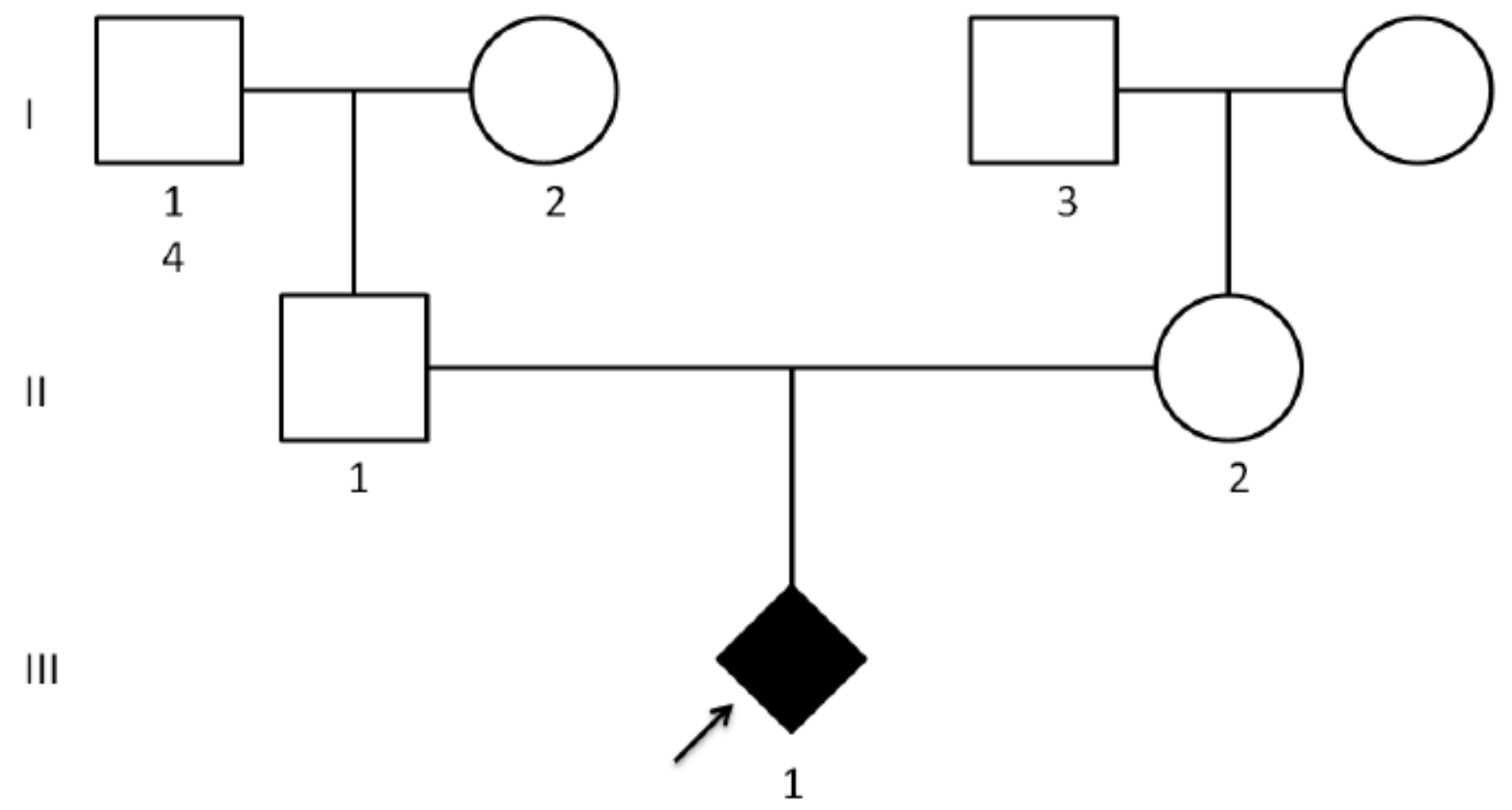

Figure 1. The pedigree of the present family. Open symbols represent healthy individuals. Squares indicate males, circles indicate females, black rhombus indicate affected fetus. The proband is indicated by an arrow.

\section{Figure 1}

See image above for figure legend. 

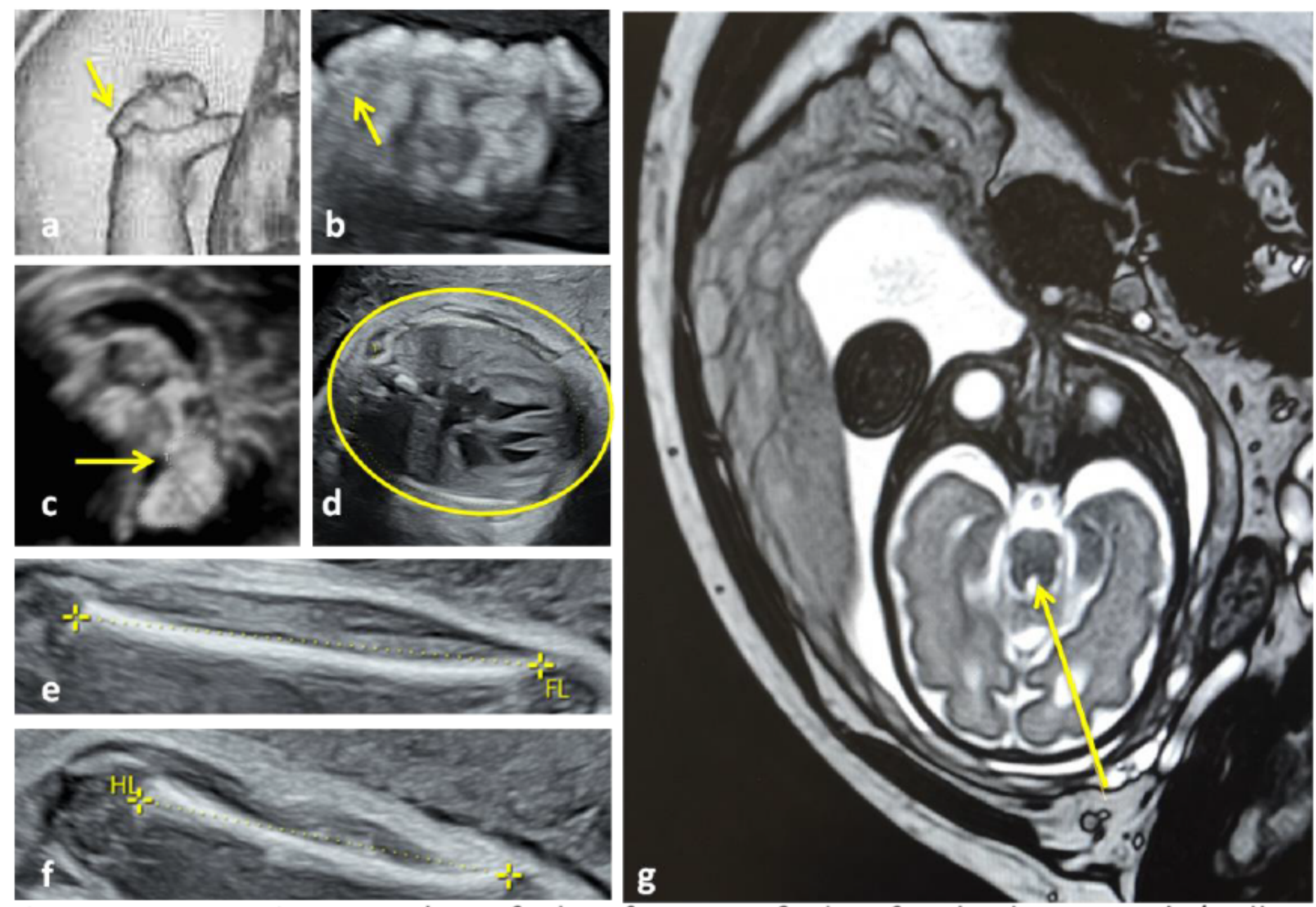

Figure 2. Imaging results of the fetus. a-f the fetal ultrasound (Yellow arrowheads indicated: a-b polydactyly of the fetal right hand; $\mathbf{c}$ the deformed cerebellar vermis; $\mathbf{d}$ restricted thoracic cage; e shorter FL and $\mathbf{f}$ shorter $\mathrm{HL}$ ); $\mathbf{g}$ the fetal MRI (Yellow arrowheads showed the thick and long superior cerebellar peduncles, forming the "molar tooth sign").

Figure 2

See image above for figure legend. 
a

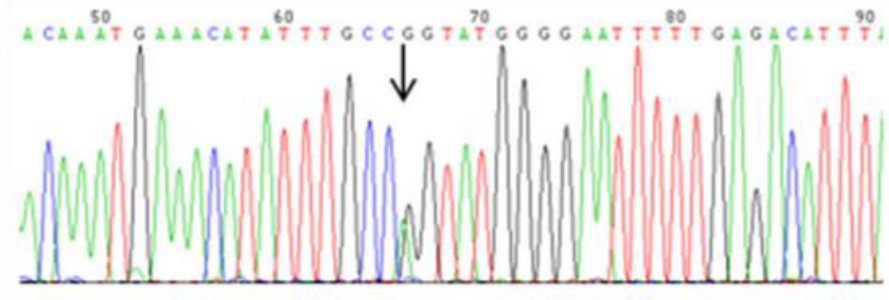

b

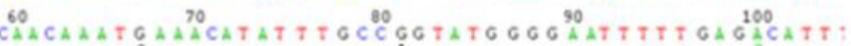

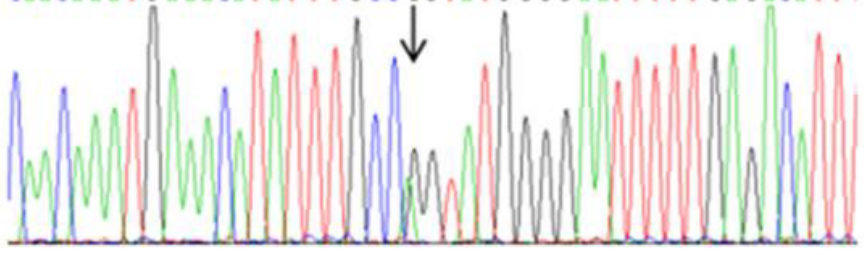

C

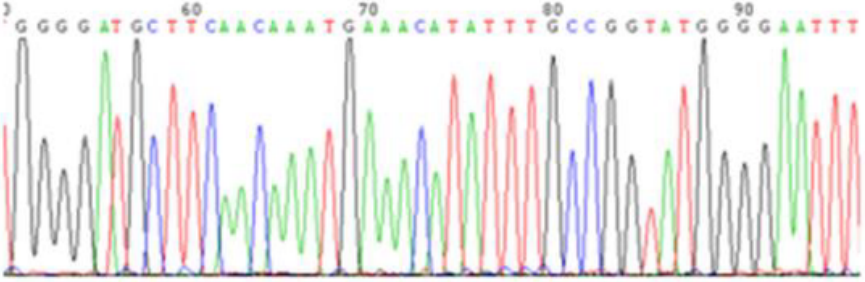

d

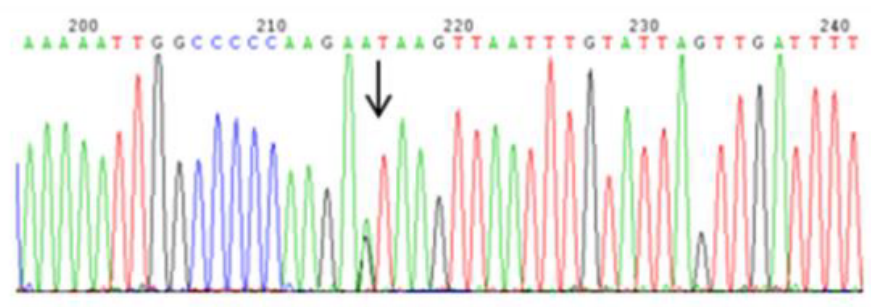

e
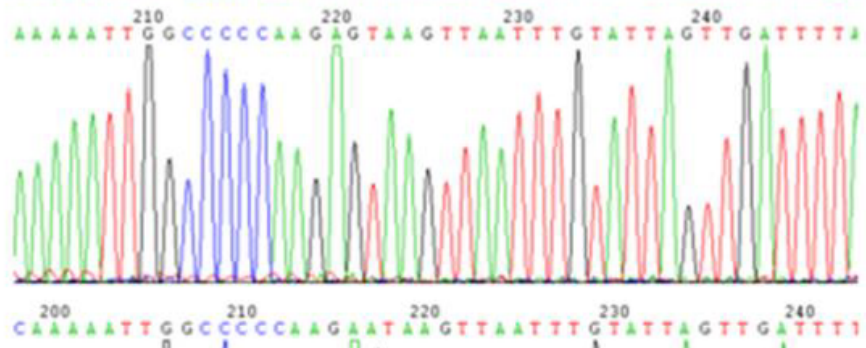

f

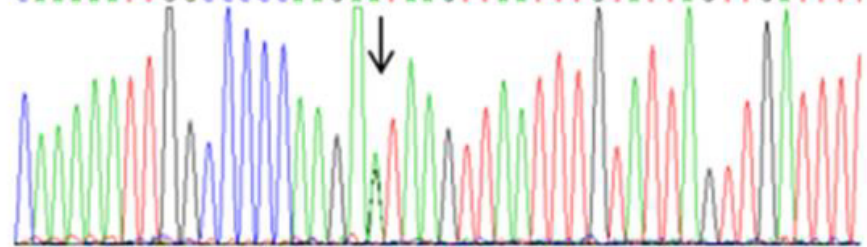

Figure 3. Sanger sequences of the compound heterozygous KIAA0586 mutations. a,d Compound heterozygous KIAA0586 mutations (c.3303G>A and c.3940+1G>A) in the fetus; b,c The synonymous mutation (c.3303G $>A$ ) deteced in the pregnant woman but not in fetus' father; e,f The novel canonical splice site mutation (c.3940+1G $>$ A) detected in the fetus' father but not in the pregnant woman. (Black arrow indicates the mutations)

Figure 3

See image above for figure legend. 

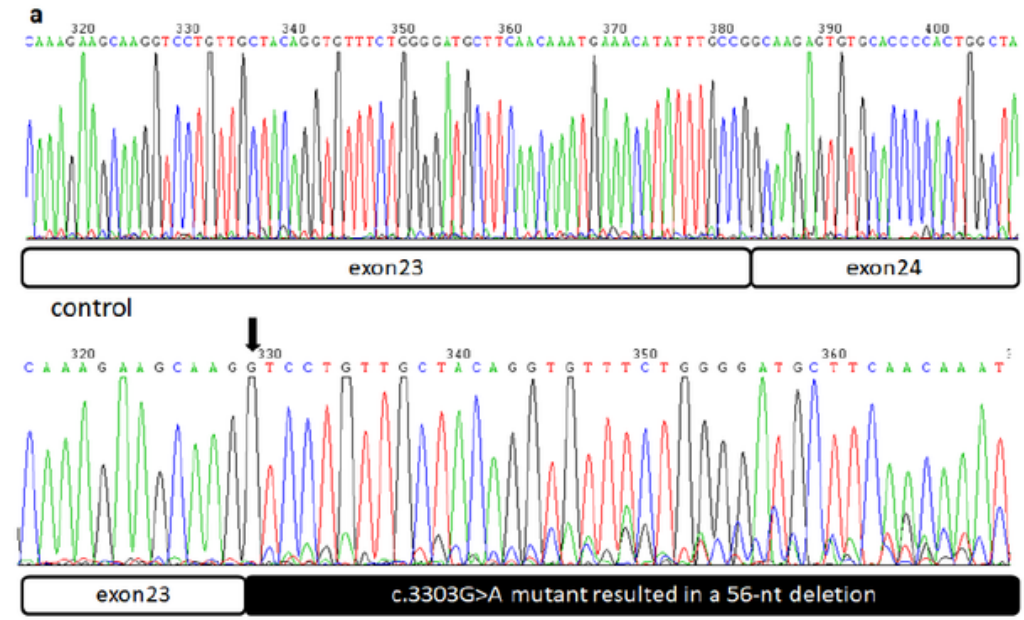

c.3303G $>$ A mutant

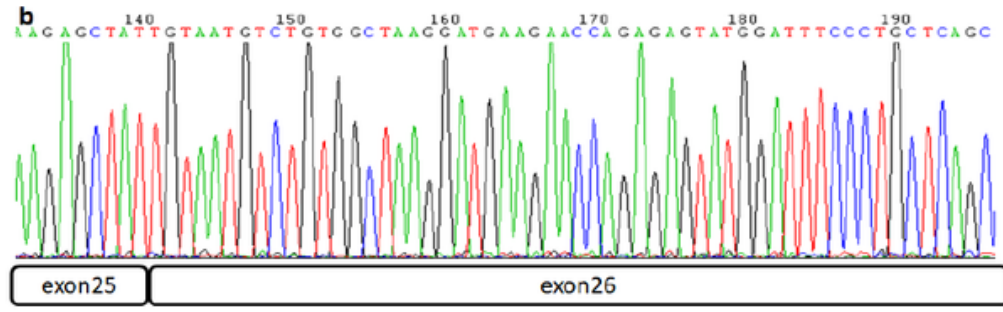

control

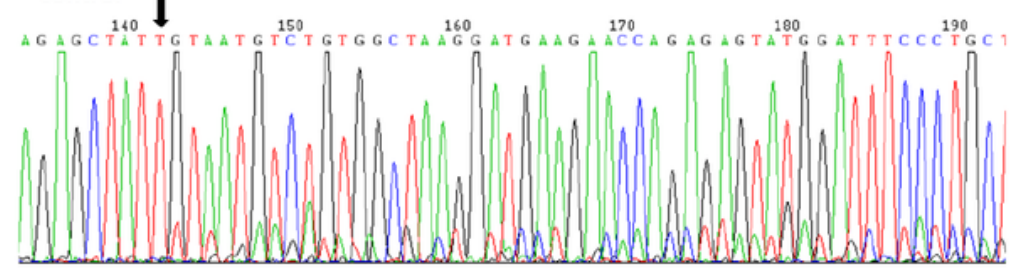

exon25 c.3940+1G>A mutant resulted in exon 26 skipping

c. $3940+1 \mathrm{G}>$ A mutant

c

No Mutation

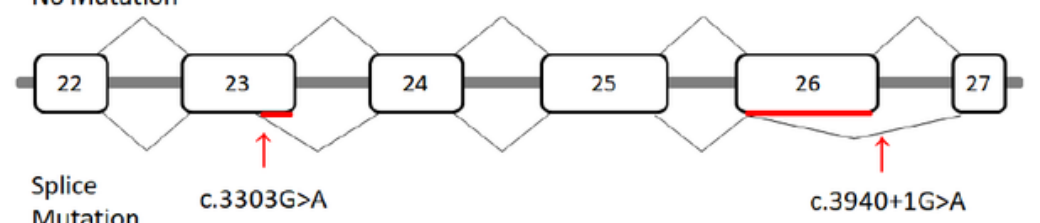

Figure 4. Molecular results. a Sanger sequencing for the synonymous-near-splice mutation (c.3303G>A) resulting in a 56-nt deletion. (Black arrow indicates the starting position of the aberrant sequence) $b$ Sanger sequencing for the canonical splice site mutation (c.3940+1G>A), resulting in exon 26 skipping. (Black arrow indicates the starting position of the aberrant sequence) c Schematic representation of the consequences of the mutations in KIAA0586 gene. (Red bars in exon 23 and 26 indicate the $56 \mathrm{bp}$-sequence and exon 26 removed by the splice site mutations, respectively)

\section{Figure 4}

See image above for figure legend. 